\title{
Theoretical and experimental study of a wireless power supply system for moving low power devices in ferromagnetic and conductive medium
}

\author{
Salaheddine Safour and Yves Bernard* \\ GeePs Laboratory, CENTRALESUPELEC-CNRS-UPSud-UPMC, Gif-sur-Yvette, France
}

Received: 10 November 2016 / Received in final form: 2 May 2017 / Accepted: 22 September 2017

\begin{abstract}
This paper focuses on the design of a wireless power supply system for low power devices (e.g. sensors) located in harsh electromagnetic environment with ferromagnetic and conductive materials. Such particular environment could be found in linear and rotating actuators. The studied power transfer system is based on the resonant magnetic coupling between a fixed transmitter coil and a moving receiver coil. The technique was utilized successfully for rotary machines. The aim of this paper is to extend the technique to linear actuators. A modeling approach based on 2D Axisymmetric Finite Element model and an electrical lumped model based on the two-port network theory is introduced. The study shows the limitation of the technique to transfer the required power in the presence of ferromagnetic and conductive materials. Parametric and circuit analysis were conducted in order to design a resonant magnetic coupler that ensures good power transfer capability and efficiency. A design methodology is proposed based on this study. Measurements on the prototype show efficiency up to $75 \%$ at a linear distance of $20 \mathrm{~mm}$.
\end{abstract}

\section{Introduction}

Nowadays, mechatronic systems become highly automated due to the increasing use of the artificial intelligence. Such systems require additional information regarding the environment in which they operate; therefore, significant number of sensors is utilized to measure different physical quantities that will be used to supervise, control or diagnostic the state of the full system. The increasing demand at the system level to increase the reliability and to decrease the maintenance time involves each component or module of the mechatronic system.

This paper focuses on the power supply module for moving sensors in linear and rotating actuators (Fig. 1a) which could be part of a mechatronic system. The power supply of the sensors could be achieved by simple direct cable, battery or wireless solution. Recently energy harvesting based solutions for low power consumption systems arises [1-5]. The selection of the technique depends in many factors: required power, life time, mobility constraint, accessibility and maintainability. The battery based solution is not preferred in many applications; their replacement is expensive over the sensor lifetime. In this study, wireless solution based on magnetic coupling technique was selected in order to increase the system life time and reliability. The technique was utilized successfully for rotary machines [6-8]. The aim of this paper is to

\footnotetext{
* e-mail: yves.bernard@u-psud.fr
}

extend the technique to linear actuator by taking into consideration the variable distance between the coils (Fig. 1b) in conductive and ferromagnetic environment.

Figure $1 \mathrm{~b}$ shows a high-level bloc diagram of a complete wireless power supply module assuming an external DCBus as the available energy source and a DC-voltage required by the low power device. In this study, we targeted a $500 \mathrm{~mW}$ power transfer to the device, which covers most of the current sensors in the market. The sensor is modeled by an equivalent resistance $R_{S}=300 \Omega$ that was calculated based on the power consumption and voltage. The design of the wireless power supply system has to take into consideration two main constraints: the geometry and the limited available space.

The resonant magnetic coupling utilizes two coils: transmitter and receiver. The large air gap between the coils leads to a poor magnetic coupling coefficient $k$ defined by equation (1) where $\phi_{11}, \phi_{22}$ are the self-magnetic flux and $\phi_{12}, \phi_{21}$ are the mutual magnetic flux.

$$
k=\sqrt{\frac{\phi 12}{\phi 11} \frac{\phi 21}{\phi 22}} .
$$

Operating the system in resonance mode allows the increase of the power transfer capability and the enhancement of the system efficiency while ensuring the equipment sizes remain manageable $[9,10]$. The resonance is achieved by connecting capacitors to the transmitter and receiver coils (Fig. 1b) and operating at the resonance frequency. With two capacitors, four compensation topologies appear $\mathrm{SS} / \mathrm{SP} / \mathrm{PS} / \mathrm{PP}$, where $\mathrm{S}$ and $\mathrm{P}$ stand, respectively, for 


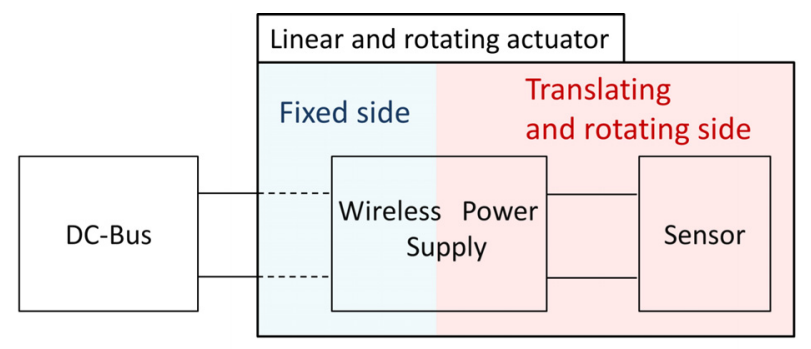

(a)

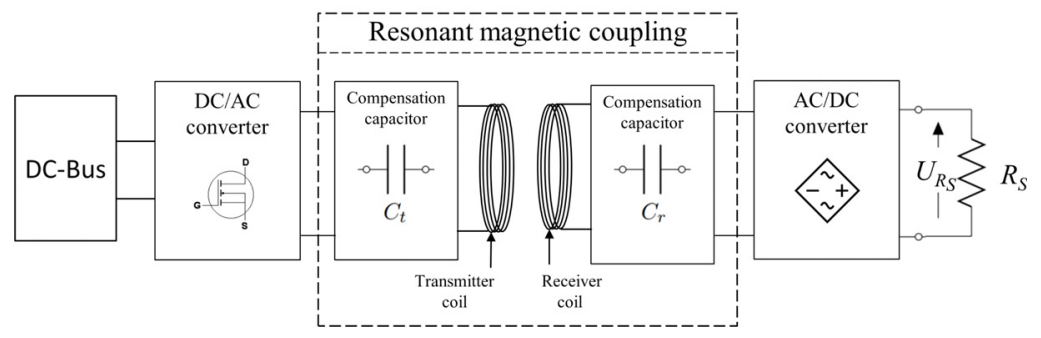

(b)

Fig. 1. (a) Wireless power supply in the linear and rotating actuator and (b) the components of the system.

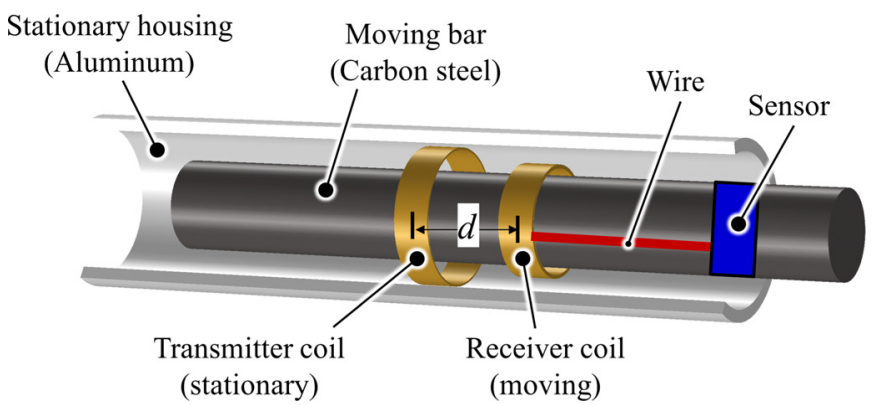

Fig. 2. Transmitter and receiver coils within the actuator.

series and parallel at the transmitter coil (first letter) and the receiver coil (second letter) [11]. The compensation topology is to be chosen according to the source (type: current or voltage, maximum amplitudes) and the load requirements (voltage or current amplitude). For instance, for current source, parallel compensation in the transmitter side will lead to high voltage across the source, which could be an issue for the switching components (exceed the maximum operating voltage).

More passive components could be connected to either the transmitter coil or the receiver coil to achieve load independent voltage or current gain [12], to increase the power transfer capability [13] or to reduce losses in the power electronics [14], however, this could add more design complexity for application with variable magnetic coupling coefficient. The coils number is another degree of freedom to enhance the power transfer distance. The use of a third intermediate resonant coil is reported in [15]. Four coils make a popular wireless power transfer architecture consisting of driver coil, transmitter coil, receiver coil and load coil [16], this architecture is space demanding and also suffers from high sensitivity to the design parameters which lead to more design complexity, such as tuning the distance between both transmitter coils (driver and transmitter coils) or tuning the

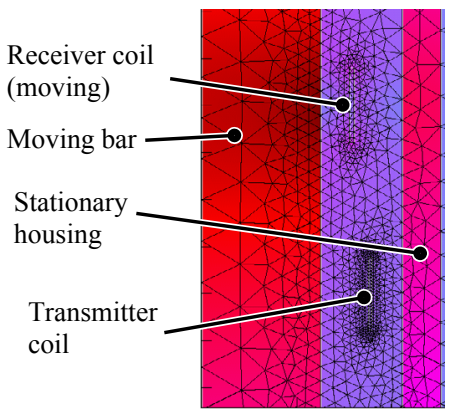

(a)

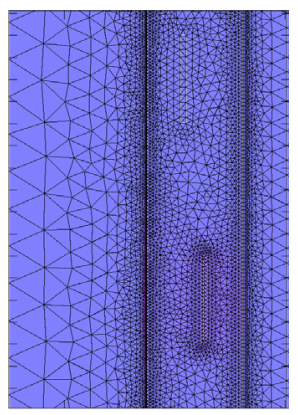

(b)
Fig. 3. Geometry meshing for (a) low frequency and (b) high frequency.

resonance frequency of the system [17].The available space, the transfer distance rate, the level of power to be transferred and the system efficiency are the main requirements for the design of such systems. This paper aims to provide a design consideration and methodology to achieve optimal design of resonant magnetic coupler for linear and rotating actuator based on the results of parametric analysis and experimental measurements.

\section{Wireless power supply system analysis}

\subsection{Studied system}

The analyzed structure (Fig. 2) is composed of moving and rotating carbon-steel bar (ferromagnetic material) and stationary aluminum housing (conductive material). The shape of these parts is assumed to be cylindrical. For the study presented in this paper we have chosen the two coils architecture for its simplicity of integration and tuning. The transmitter coil is clamped to the housing; the receiver coil that feeds the sensor is clamped to the bar. The turns 


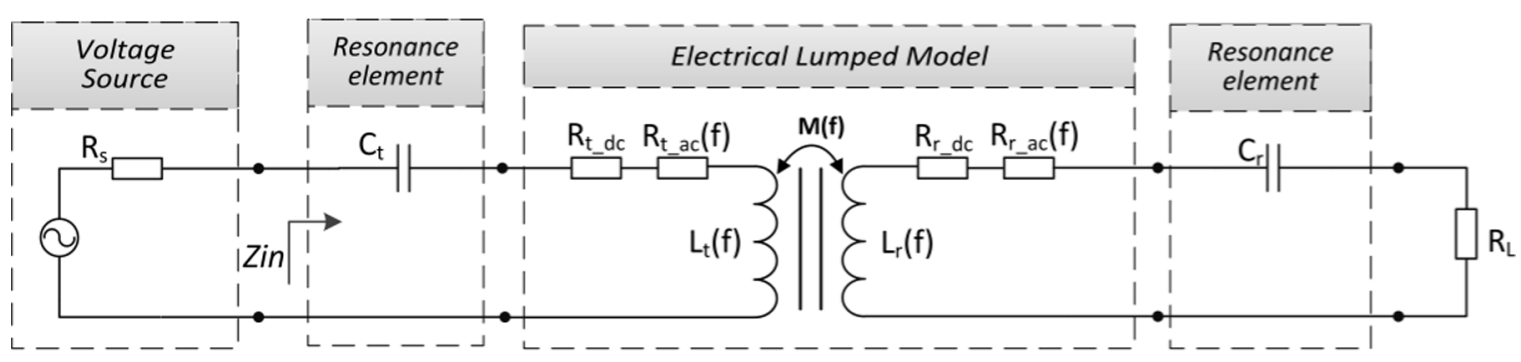

Fig. 4. Electrical lumped model of the resonant magnetic coupler.

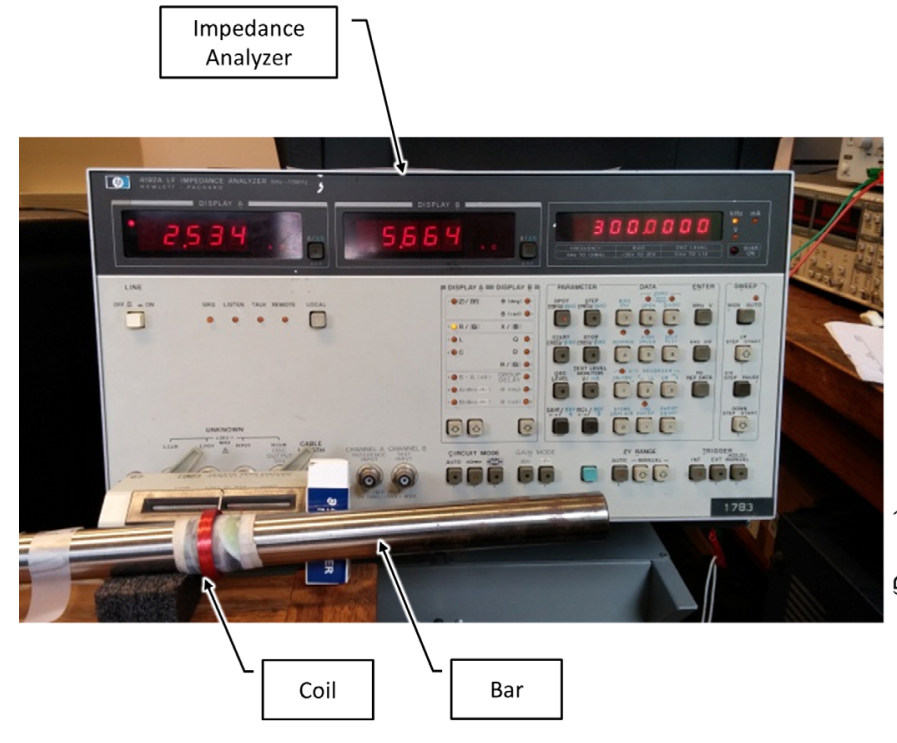

(a)
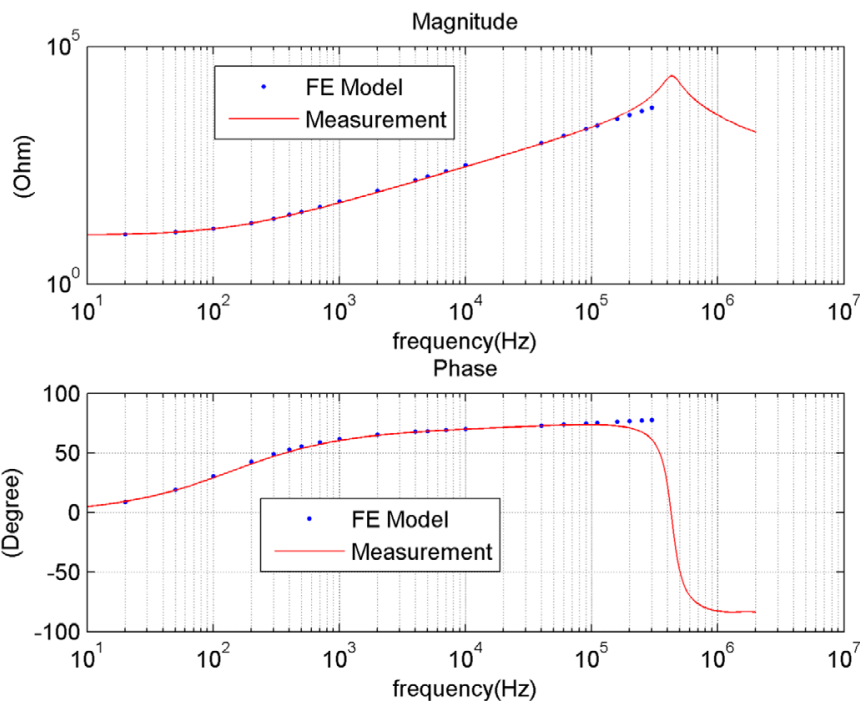

(b)

Fig. 5. (a) $\mu$-identification setup and (b) simulated and measured impedance of the coil.

number $\left(N_{T}=20, N_{R}=40\right)$ of the coils was guided by the available space constraints and cost (minimum turns number). Litz wire is used to reduce skin depth and proximity effects. The transmitter and the receiver coil have, respectively, 14.5 and $18.6 \mathrm{~mm}$ outer radius. The power transfer range $d$ defined by the distance between the middle section plane of coils varies between 0 and $20 \mathrm{~mm}$.

To achieve the resonance the series-series topology was adopted since we have a voltage source (DC-Bus) at the transmitter side and a capacitive filter at the receiver side with a constant load (the sensor).

\subsection{Modeling approach}

A system modeling approach that combines a Finite Element (FE) model and an electrical lumped model is introduced in order to analyze the effect of the surrounding materials, the resonance topology and the load on the performance of the wireless power supply system. The analysis of the results obtained with this approach will give guidance to ensure an effective design.

The structure is analyzed using FE method based software (COMSOL Multiphysics). The problem was reduced to $2 \mathrm{D}$ axisymmetric and the magneto-quasistatic equations were solved in the frequency domain. The constitutive relations were assumed to be linear. The hysteresis losses in the materials were modeled by a complex magnetic permeability (Eq. (2)). The residual losses of the materials, the skin and proximity effects in the coils were not taken into account. The winding is modeled by a rectangular shape with uniform current density.

$$
\mu^{*}=\mu^{\prime}+j \mu^{\prime \prime}
$$

The surrounding materials are ferromagnetic or conductive; therefore, the skin depth varies with respect to the frequency. In order to well take into account this effect while respecting a compromise between the error on the simulation results and time of calculation, dynamic meshing is achieved by updating the setting of the meshing in the FE model at each frequency using a MATLAB code based on the skin depth calculation (Fig. 3).

On another hand, a MATLAB routine that includes: electrical lumped model of the magnetic part, resonance elements, voltage source and resistive load (Fig. 4) is achieved in order to assess the influence of each element on the whole system performance (active/reactive power, efficiency, resonance frequency) within a frequency range under the assumption of sinusoidal voltage source. 


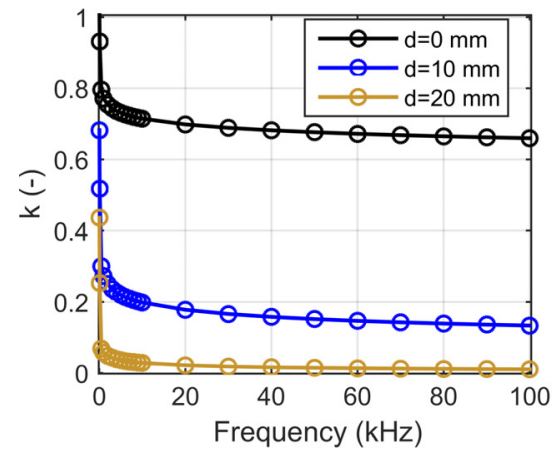

(a)

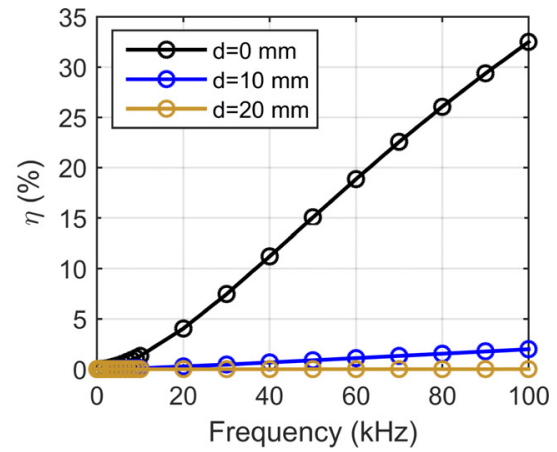

(b)

Fig. 6. Numerical results of (a) magnetic coupling coefficient and (b) system efficiency $\left(R_{L}=100 \Omega\right.$, system operating at resonance at all frequencies) as a function of frequency.

The values of the following electrical lumped model parameters are derived from the FE model simulation results:

- $R_{t \quad d c}, R_{r \quad d c}$ the coil resistance;

$-R_{t_{-} a c}^{-}(f), R_{r_{-} a c}(f)$ the equivalent series resistance with frequency dēpendence to take into account losses in the surrounding materials;

- $L_{t}(f), L_{r}(f)$ the coils self-inductance;

- $M(f)$ the mutual inductance.

In order to achieve circuit analysis, the two-port network method was adopted due to its flexibility (e.g. adding components, changing their connection (series or parallel)). Each component of the circuit in Figure 4 is considered as a two-port network and modeled with an $A B C D$-parameters matrix given by:

$$
\left[\begin{array}{l}
V_{1} \\
I_{1}
\end{array}\right]=\left[\begin{array}{ll}
a_{11} & a_{12} \\
a_{21} & a_{22}
\end{array}\right]\left[\begin{array}{l}
V_{2} \\
-I_{2}
\end{array}\right]=\left[\begin{array}{ll}
A & B \\
C & D
\end{array}\right]\left[\begin{array}{l}
V_{2} \\
-I_{2}
\end{array}\right],
$$

where $\quad a_{11}=\left.\frac{V_{1}}{V_{2}}\right|_{I_{2}=0} ; \quad a_{12}=-\left.\frac{V_{1}}{I_{2}}\right|_{V_{2}=0} ; \quad a_{21}=\left.\frac{I_{1}}{V_{2}}\right|_{I_{2}=0} ;$ $a_{22}=-\left.\frac{I_{1}}{I_{2}}\right|_{V_{2}=0}$.

For instance, the two port network of the magnetic coupler is described first by the following $z$-parameters matrix:

$$
\begin{aligned}
& {\left[\begin{array}{l}
V_{1} \\
V_{2}
\end{array}\right]=\overbrace{\left[\begin{array}{ll}
z_{11} & z_{12} \\
z_{21} & z_{22}
\end{array}\right]}^{Z_{\text {coupler }}}} \\
& \quad=\left[\begin{array}{cl}
R_{t_{d} c}+R_{t_{a} c}+j \omega L_{t} & j \omega M \\
j \omega M & \left(R_{r_{d} c}+R_{r_{a} c}+j \omega L_{r}\right)
\end{array}\right]\left[\begin{array}{l}
I_{1} \\
I_{2}
\end{array}\right] .
\end{aligned}
$$

Then the $A B C D$-parameters matrix is derived from the $z$ parameters matrix (Eq. (4)) using the interrelations formula:

$$
\left[\begin{array}{l}
V_{1} \\
I_{1}
\end{array}\right]=\frac{1}{z_{21}}\left[\begin{array}{ll}
z_{11} & z_{11} z_{22}-z_{12} z_{21} \\
1 & z_{22}
\end{array}\right]\left[\begin{array}{l}
V_{2} \\
-I_{2}
\end{array}\right]
$$

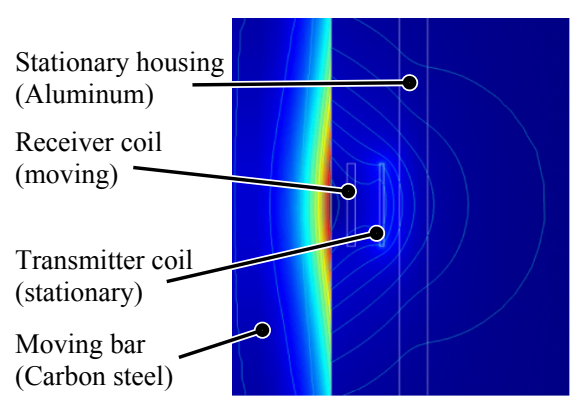

(a)

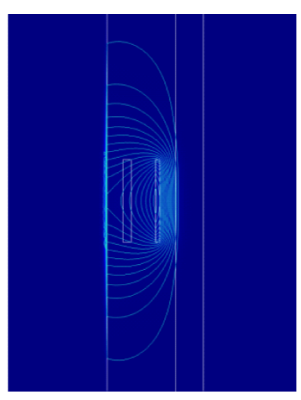

(b)
Fig. 7. Simulation results of the magnetic field lines distribution at $50 \mathrm{~Hz}$ (a) and $20 \mathrm{kHz}(\mathrm{b})$.

The method allows accessing multiple quantities: voltage gain, current gain, circuit input impedance, input power, output power and efficiency. The calculations of those quantities are derived using symbolic mathematics software (Maxima) based on the two-port network matrices.

\subsection{Carbon steel $\mu_{r}$ identification}

The FE model requires the electromagnetic properties of each part in the simulation setup. The value of the electrical conductivity of the carbon steel and the aluminum is, respectively, $\sigma_{b}=6 \times 10^{6} \mathrm{~S} / \mathrm{m}$ and $\sigma_{h}=4$ $\times 10^{7} \mathrm{~S} / \mathrm{m}$, however, the relative magnetic permeability of the carbon steel was estimated in small-signal domain using a coil and a carbon-steel bar on its center. The value $\mu_{b}=100$ was obtained by minimizing the error between the impedance spectrum obtained by measurement using an impedance analyzer and the one obtained by FE simulation (Fig. 5).

\subsection{Resonant magnetic coupling limitation}

In a series-series resonant magnetic coupling, when operating at the resonance frequency $f_{0}$ at which the coils reactances are compensated by the capacitors reactances, 


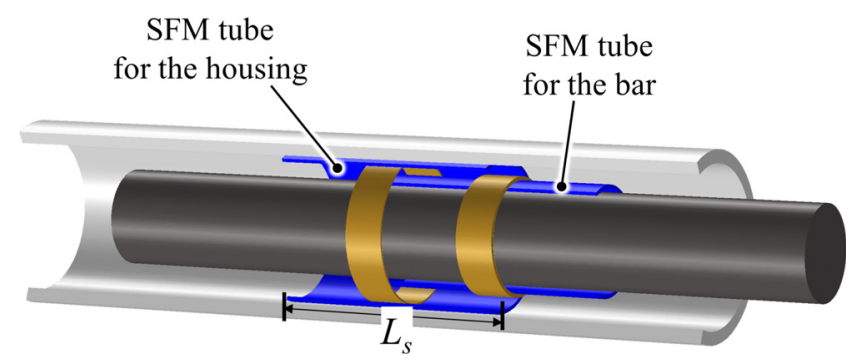

Fig. 8. 3-D view of the magnetic coupler with the two SFM based tubes.

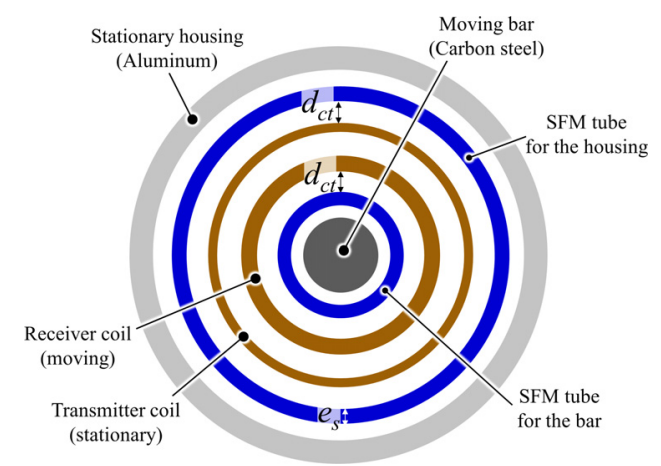

Fig. 9. Cross section view of the magnetic coupler with the two SFM based tubes.

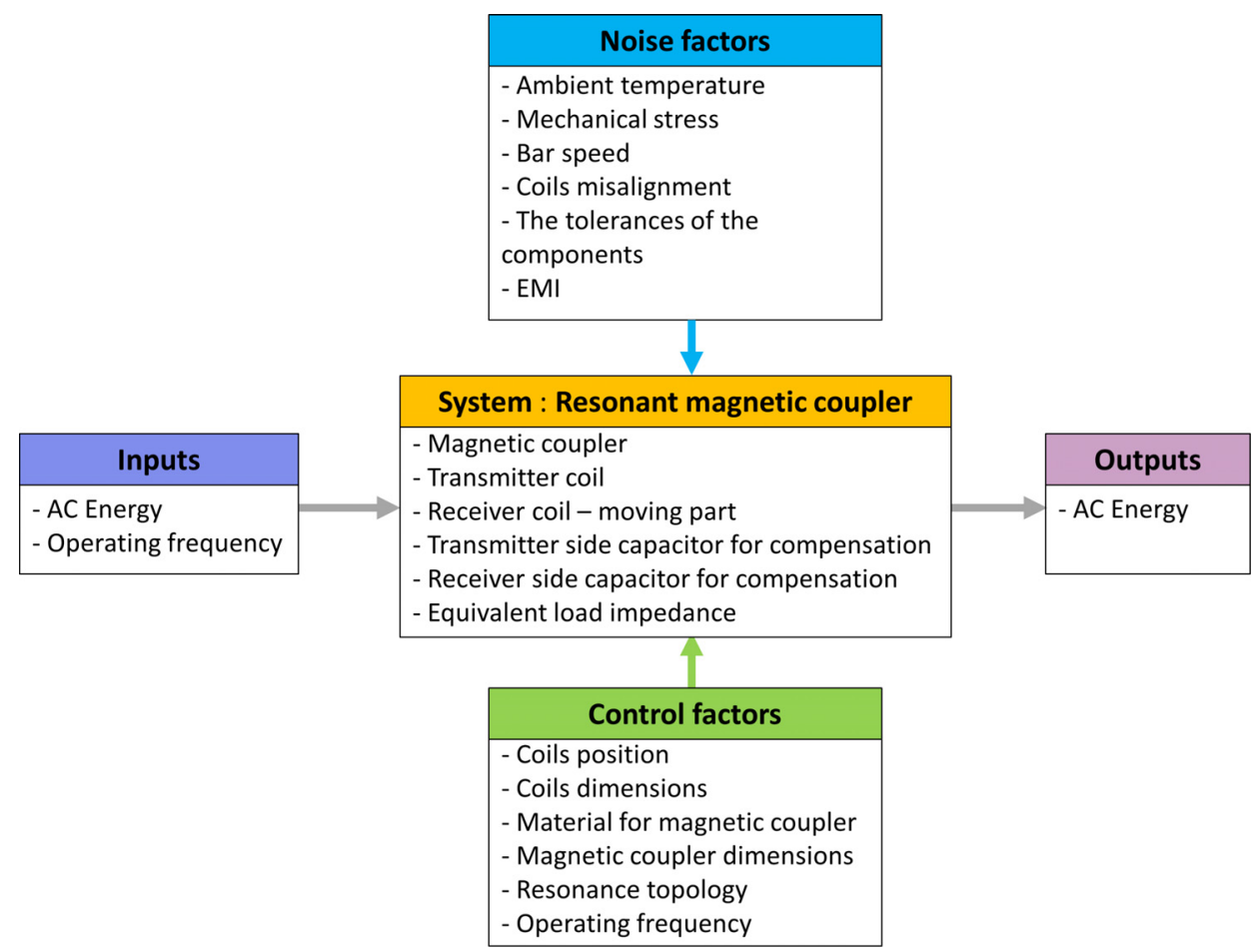

Fig. 10. Parameter diagram of the resonant magnetic coupler.

the obtained system efficiency ( for $R_{s}=0 \Omega$ ) is given by:

$$
\eta=\frac{\lambda}{\lambda+1} \frac{k^{2} Q_{t} Q_{r}}{1+\lambda+k^{2} Q_{t} Q_{r}},
$$

where $\lambda={ }_{R_{L}} / R_{R_{r}} ; Q_{t}={ }^{L_{t}} \omega_{0} / R_{t} ; Q_{r}={ }_{L_{r}} \omega_{0} / R_{R_{r}}$.

Equation (6) is obtained by calculation using the electrical lumped model; it shows that the magnetic circuit of the wireless power supply system could be characterized by two quantities: the magnetic coupling factor $(k)$ and the quality factor of the coils (transmitter $Q_{t}$ and receiver $Q_{r}$ ) [18].

The FE simulation results obtained for the structure in Figure 2 shows that the weak magnetic coupling (Fig. 6a) combined with the high losses in different components lead to poor system efficiency (Fig. 6b).
The reason behind the drop of the magnetic coupling for a given distance is the fact that eddy current on the carbon steel and the aluminum part repels the magnetic field at its surface (skin effect), therefore the magnetic flux on the receiver coil decreases (Fig. 7) leading to inefficient system at the maximum distance regardless of the operating frequency.

\section{Resonant magnetic coupler design and validation}

The materials (carbon steel and aluminium) from which the parts of the structure are made cannot be changed to materials with better electromagnetic properties due to some mechanical design constraints. In order to address the issue, a new structure based on two tubes made of soft 


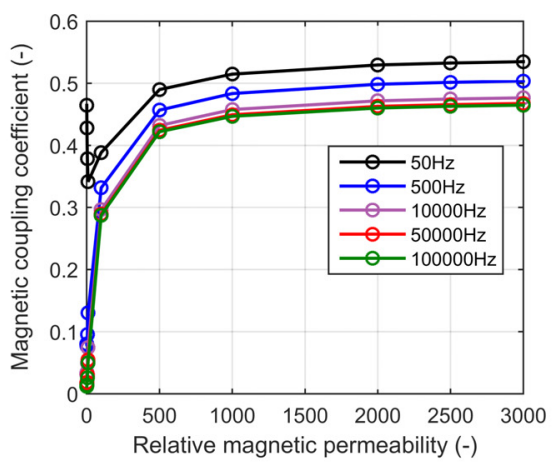

(a)

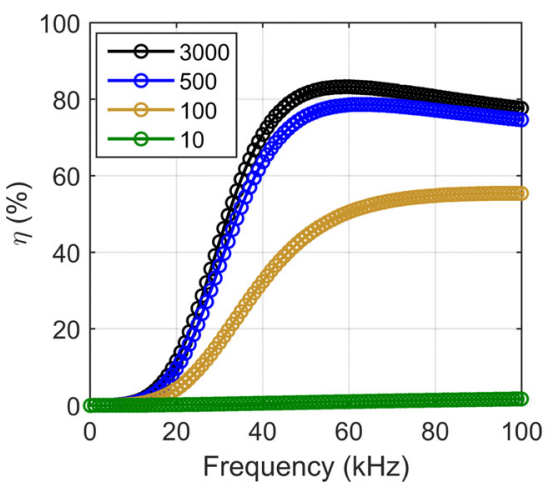

(c)

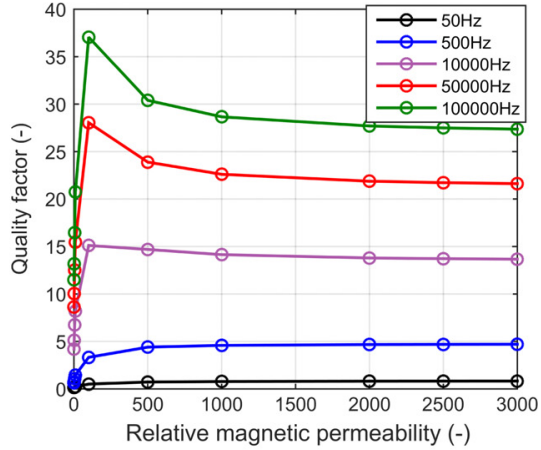

(b)

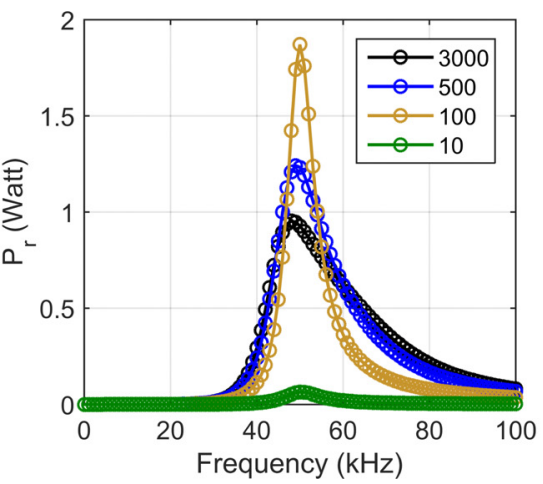

(d)

Fig. 11. Simulation results of (a) magnetic coupling coefficient, (b) transmitter side quality factor as a function of the relative magnetic permeability of the SFM tubes at different frequencies, (c) efficiency and $(\mathrm{d})$ received power $\left(f_{\text {res }}=50 \mathrm{kHz}\right.$, $R_{L}=100 \Omega, V_{\text {in }}=5 \mathrm{~V}$ ) for different relative magnetic permeability.

ferromagnetic material (SFM) with very low electrical conductivity is proposed. The added tubes with the length $L_{s}$ will canalize the magnetic field from the transmitter to the receiver coil (Fig. 8). The first one is clamped to the bar and the second to the housing.

Figure 9 shows a cross section of the proposed structure showing two design dimensions:

$-d_{c t}$ is the distance between the SFM tube and the bar or housing;

$-e_{s}$ is the thickness of the SFM tubes.

To analyse the new structure, the worst configuration was considered ( $20 \mathrm{~mm}$ as maximal transfer distance). The resonance operating mode is achieved by tuning the capacitors $C_{t}$ and $C_{r}$ using a simple resonant $L C$ circuit formula:

$$
C_{t}=\frac{1}{L_{t} \omega_{r e s}^{2}} \quad \text { and } \quad C_{r}=\frac{1}{L_{r} \omega_{r e s}^{2}}
$$

The parameter diagram in Figure 10 summarizes the different factors that might have an impact on the performance of the resonant magnetic coupling. The noise factors are out of the scope of this paper, only some of the control factors are analyzed in the next section in order to give guidance for optimal design.

\subsection{Parametric analysis}

In this section, we analyse the effect of the properties of the SFM tubes and their dimensions on the resonant magnetic coupling performance. To reduce the number of the degrees of freedom, the coils dimensions have been chosen based on the maximum available space and positioned in a manner that their revolution axes match the ones of the other parts (Fig. 9). It would be also possible to position the coils in a manner that their revolution axes are perpendicular to the lateral surface of the SFM tubes.

First, the effect of the relative magnetic permeability $\left(\mu_{r}\right)$ was analyzed. Figure 11a shows that at low frequency $(50 \mathrm{~Hz})$ increasing slightly $\mu_{r}$ through a value of 50 decreases the magnetic coupling coefficient, exceeding this value, the magnetic coupling coefficient increases. This behaviour is due to the effect of the eddy current in the ferromagnetic and conductive parts. At high frequencies, the magnetic coupling coefficient increases rapidly with the relative magnetic permeability then slowly after the value of 500. The system efficiency is enhanced and the received power is maximal at the resonance frequency $(50 \mathrm{kHz})$ when increasing the relative magnetic permeability, this is due to the fact that the added tubes concentrate the magnetic flux without suffering from high losses or skin effect (very low electrical conductivity) at the studied frequencies. 


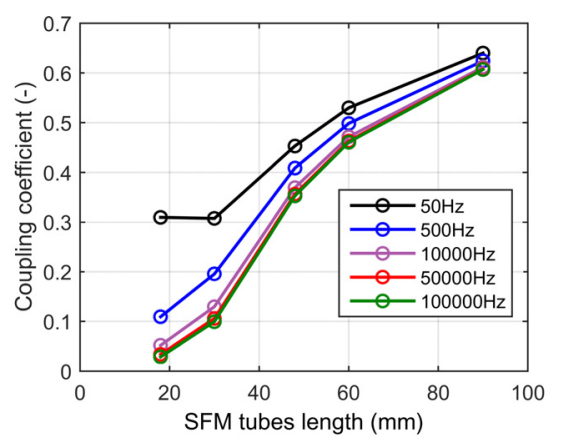

(a)

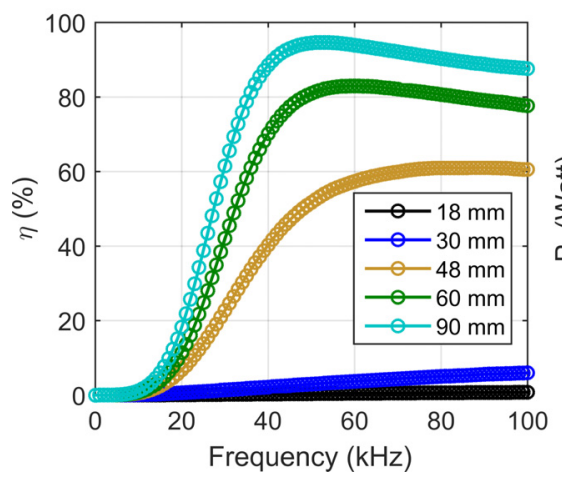

(c)

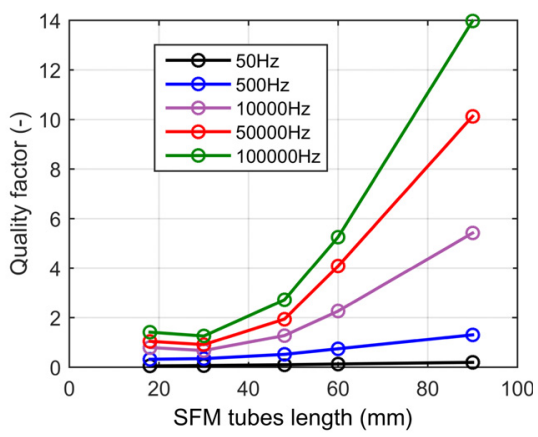

(b)

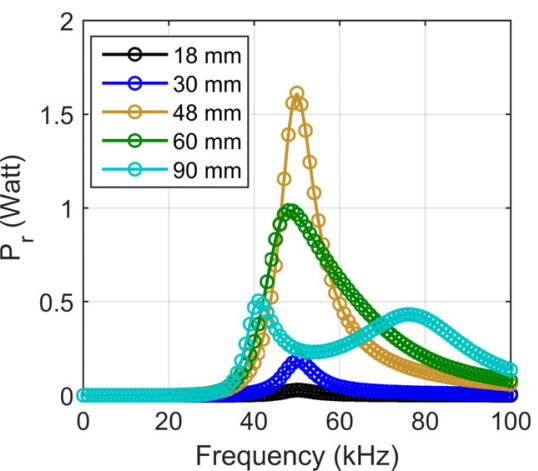

(d)

Fig. 12. Simulation results of (a) magnetic coupling coefficient, (b) transmitter side quality factor as a function of the SFM tubes length at different frequencies, (c) efficiency and (d) received power $\left(f_{\text {res }}=50 \mathrm{kHz}, R_{L}=100 \Omega\right)$ for different SFM tubes length $\left(\mu_{r}=2000, e_{s}=1 \mathrm{~mm}, d_{c t}=0 \mathrm{~mm}\right)$.

Increasing the length of the SFM tubes, increases the magnetic coupling coefficient and the quality factor for a given operating frequency (Fig. 12a and b). Good system efficiency is obtained when the length is higher than $60 \mathrm{~mm}$ $\left(L_{s}>3 d\right)$. High magnetic coupling coefficient (obtained for $L_{s}=90 \mathrm{~mm}$ ) leads to a bifurcation phenomenon that appears in the received power (Fig. 12d) with two locals maximum.

The magnetic coupling increases when increasing the thickness of the SFM tubes (on the bar and the housing) and the magnetic flux density decreases, which helps to avoid saturation in the material. Good performance and low magnetic flux density are obtained for thicknesses higher than $0.5 \mathrm{~mm}$ (Fig. 13).

Decreasing the distance between the SFM tube and the bar impact slightly the performance of the system. Null distance achieves the best performance (Fig. 14).

\subsection{Proposed design}

The parametric analysis provides guidance to design the SFM tubes: the length should be three times higher than the maximal required power transfer distance, the thickness higher than $0.5 \mathrm{~mm}$ and null distance between the coils and the tubes is preferred. Table 1 shows the material properties and dimensions of the SFM tubes chosen based on cost, available space, operating frequency, flux density level and temperature.

Simulation results show that the proposed design of the resonant magnetic coupler increases the magnetic coupling and achieves high system efficiency (Fig. 15a and b). The case where the system is tuned to operate at a resonance frequency of $50 \mathrm{kHz}$ is shown in Figure $15 \mathrm{c}$ and $\mathrm{d}$, the frequency corresponding to the maximum received power point changes with the distance between the coils.

The proposed design is capable of achieving $\sim 500 \mathrm{~mW}$ at all distances with efficiency of $\sim 80 \%$. According to Figure 16, the efficiency of the system is also load dependent; therefore, the power electronics between the receiver side of the resonant magnetic coupler and the sensor should include an impedance transformation function. The level of the transferred power to the sensor could be controlled by adjusting the amplitude of the voltage source or by controlling the operating frequency. However, by adopting the first strategy the system might not be operating in resonance mode, which leads to high VA rating and switching losses. Operating at constant frequency requires stable values of the components (capacitor, inductance, SFM properties) to achieve stable resonance frequency, leading to additional cost to have low tolerances of the system components. The system shows bifurcation phenomena (multiple zero phase angle points) in the condition $d=10 \mathrm{~mm}$ and $R_{L}=10 \mathrm{k} \Omega$, we observe three zero phase angle points (Fig. 17b) within the frequency range $20-100 \mathrm{kHz}$, this could lead to system instability [19] in case of frequency closed loop control. Decreasing the load resistance $\left(R_{L}=100 \Omega\right)$ leads to the bifurcation phenomena to disappear (Fig. 17a). The frequency corresponding to zero phase angle is then unique and increases with the distance between the transmitter and the receiver coils. This frequency could be utilized as 


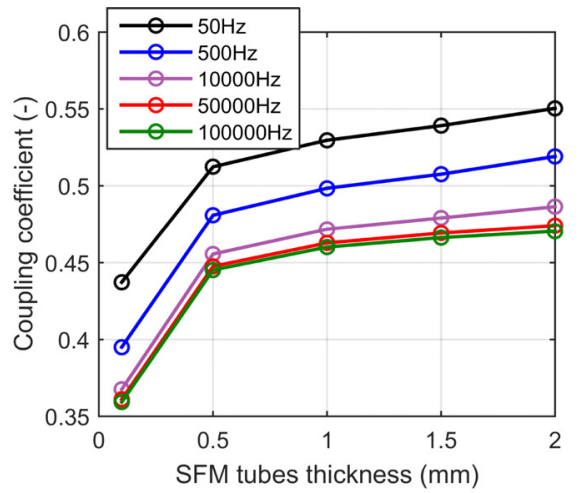

(a)

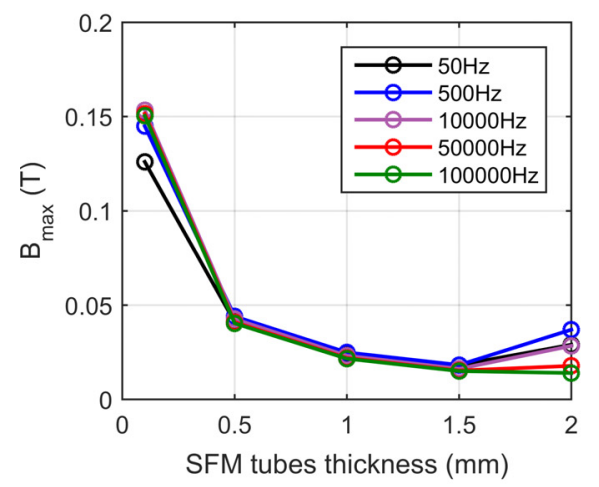

(c)

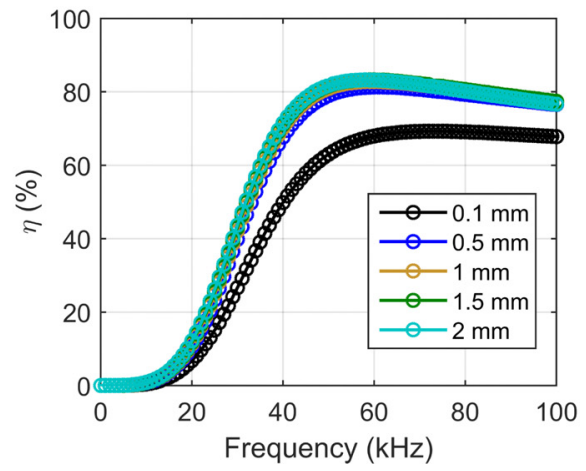

(e)

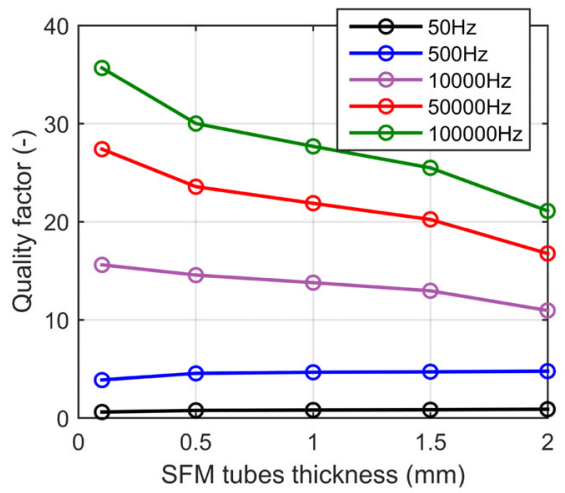

(b)

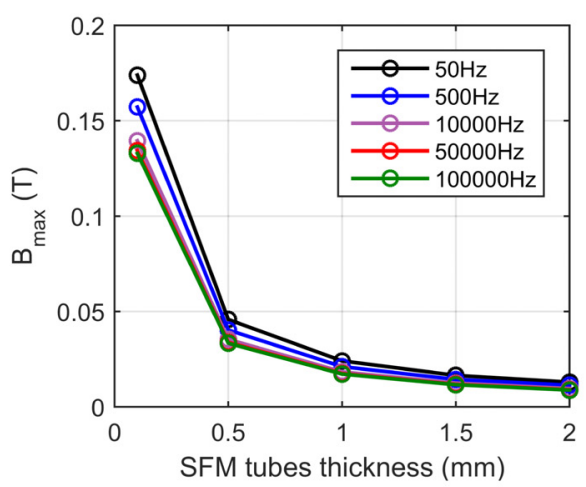

(d)

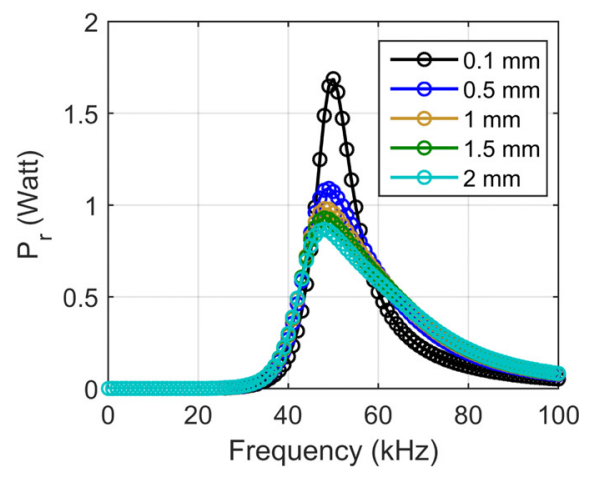

(f)

Fig. 13. Simulation results of (a) magnetic coupling coefficient, (b) transmitter side quality factor as a function of the SFM tubes thickness at different frequencies, maximal flux density in the SFM tube (c) for the bar, (d) for the housing, (e) efficiency and (f) received power $\left(f_{\text {res }}=50 \mathrm{kHz}, R_{L}=100 \Omega\right)$ for different SFM tubes thickness $\left(\mu_{r}=2000, L_{s}=60 \mathrm{~mm}, d_{c t}=0 \mathrm{~mm}\right)$.

measured quantity to achieve a 1D position sensor of the bar on which the receiver coil is clamped. Several degrees of freedom are available to avoid the bifurcation phenomena earlier in the design: load adjustment (impedance transformation), dimensions of the SFM tubes and the material properties, coils dimensions and number of turns.

\subsection{Prototype and models validation}

Figure 18 shows two pictures of the wireless power transfer prototype. The transmitter and the receiver coils are made of Litz wire in order to reduce the skin and the proximity effects. The experiment is conducted at frequencies widely below the self-resonance frequency of the coils. Multiple ceramic capacitors are connected in parallel to make the capacitors $C_{t}$ and $C_{r}$.

Experimental measurements were conducted to assess the performance of the prototype. The experimental setup (Figure 19) used a sinusoidal wave generator, linear power amplifier (HSA 4101 from NF), and an oscilloscope equipped with voltage probes and current probes capable of measuring a current as low as $1 \mathrm{~mA}$ (Hioki CT6700). The input power, output power and efficiency are processed by the oscilloscope (Keysight InfiniiVision DSO-X 3024T). 


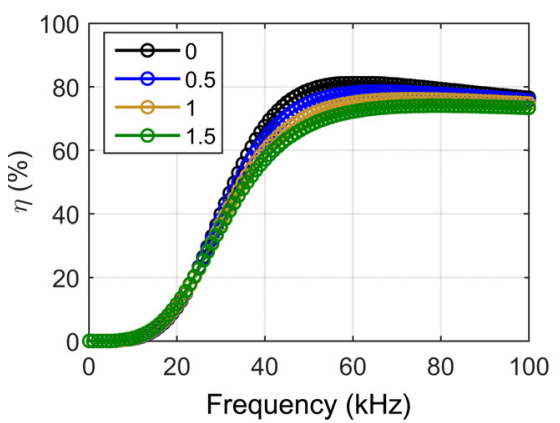

(a)

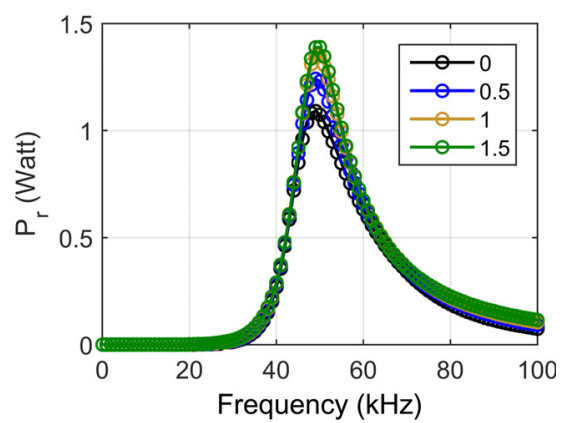

(b)

Fig. 14. Simulation results of (a) efficiency and (b) received power $\left(f_{\text {res }}=50 \mathrm{kHz}\right)$ for different distance $d_{c t}\left(\mu_{r}=2000, L_{s}=60 \mathrm{~mm}\right.$, $\left.e_{s}=0.5 \mathrm{~mm}\right)$.

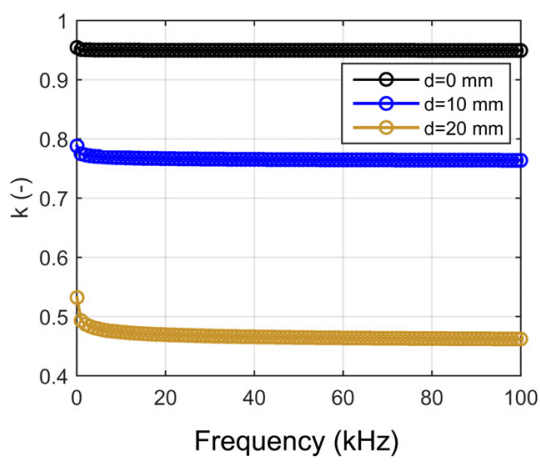

(a)

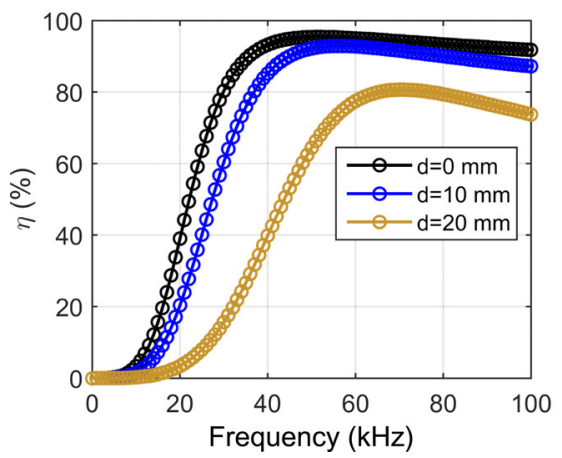

(c)

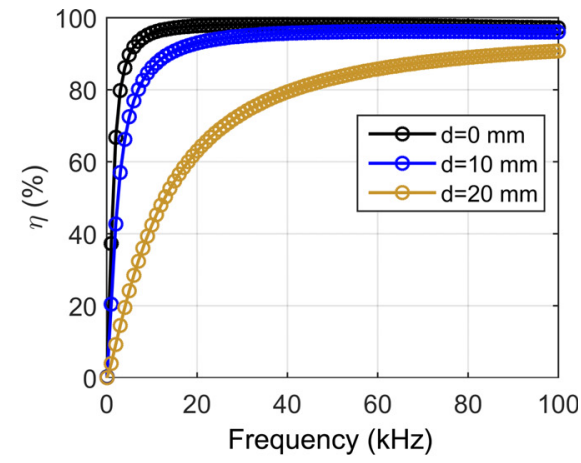

(b)

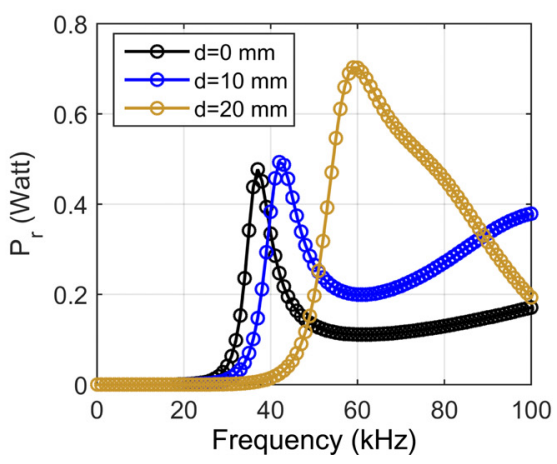

(d)

Fig. 15. Simulation results of (a) magnetic coupling coefficient and (b) system efficiency $\left(R_{L}=100 \Omega\right.$, system operating at resonance at all frequencies) as a function of frequency (c) efficiency and (d) received power $\left(f_{\text {res }}=50 \mathrm{kHz} ; R_{L}=100 \Omega\right)$ for different bar positions.

Table 1. Material properties and dimensions of the SFM tubes.

\begin{tabular}{ll}
\hline Parameter & Value \\
\hline$\mu_{r}$ & 2300 \\
$\sigma$ & $0.2 \mathrm{~S} / \mathrm{m}$ \\
$L_{s}$ & $60 \mathrm{~mm}$ \\
$e_{s}$ & $1 \mathrm{~mm}$ \\
$d_{c t}$ & $0.5 \mathrm{~mm}$ \\
\hline
\end{tabular}

In order to assess the prototype performance, the capacitors values were calculated for $F_{\text {res }}=50 \mathrm{kHz}$ using the formula $C=1 /\left(L \omega_{\text {res }}^{2}\right)$ and the self-inductances values of the coils at $d=0 \mathrm{~mm}$. Figure 20 compares the obtained experimental results using the test bench and the simulation results:

- the experimental result is labeled "Measurement";

- the simulation result using the electrical lumped model where its parameters were identified using the FE method based model is labeled "FEA+ELM";

- the simulation result using the electrical lumped model where its parameters were measured with the impedance analyzer is labeled "IA+ELM". 


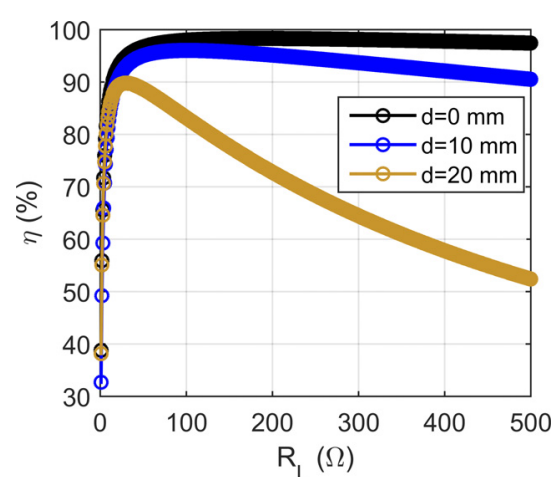

Fig. 16. Simulation result of the system efficiency as a function of the equivalent load resistance $\left(f_{\text {res }}=50 \mathrm{kHz}\right)$.
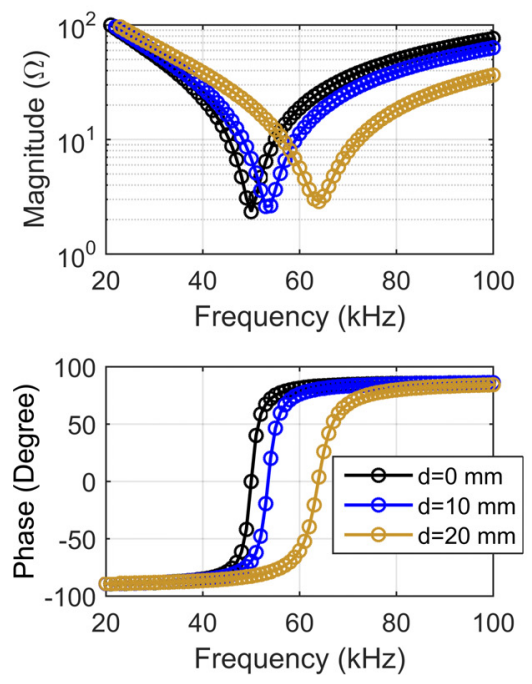

(a)

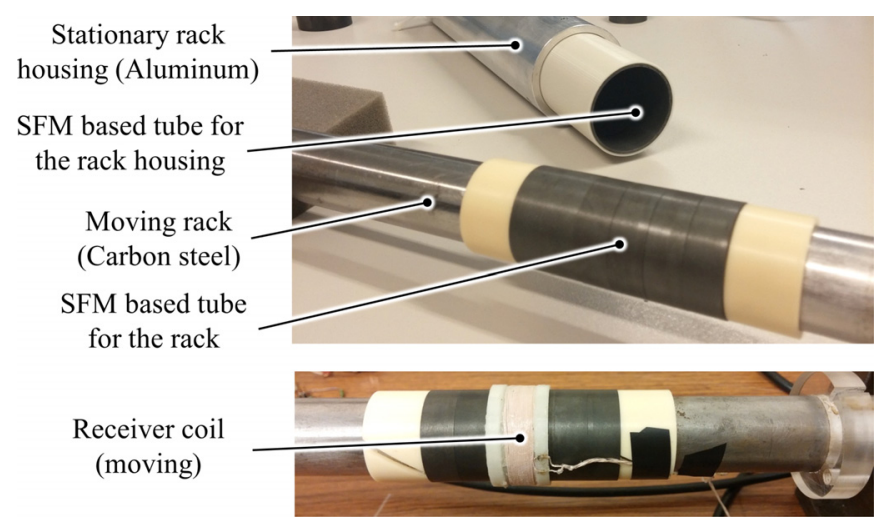

Fig. 18. Magnetic coupler prototype.
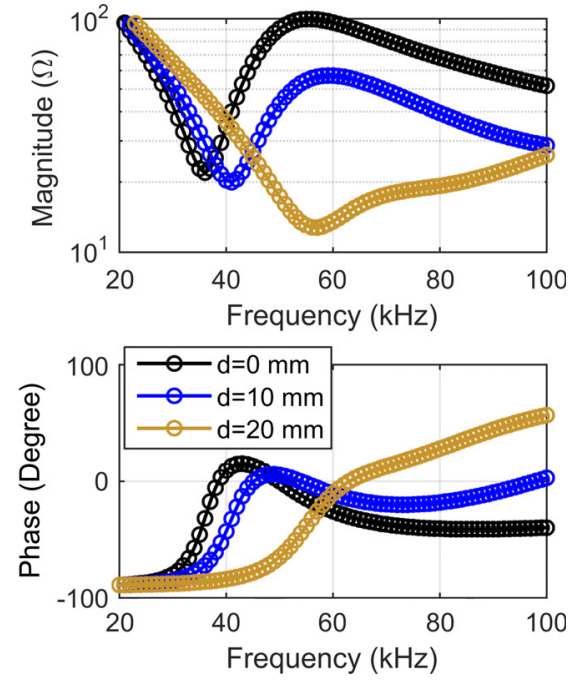

(b)

Fig. 17. Simulation results of the input impedance $\left(Z_{\text {in }}\right)$ magnitude and phase angle for (a) $R_{L}=100 \Omega$ and (b) $R_{L}=10 \mathrm{k} \Omega$.

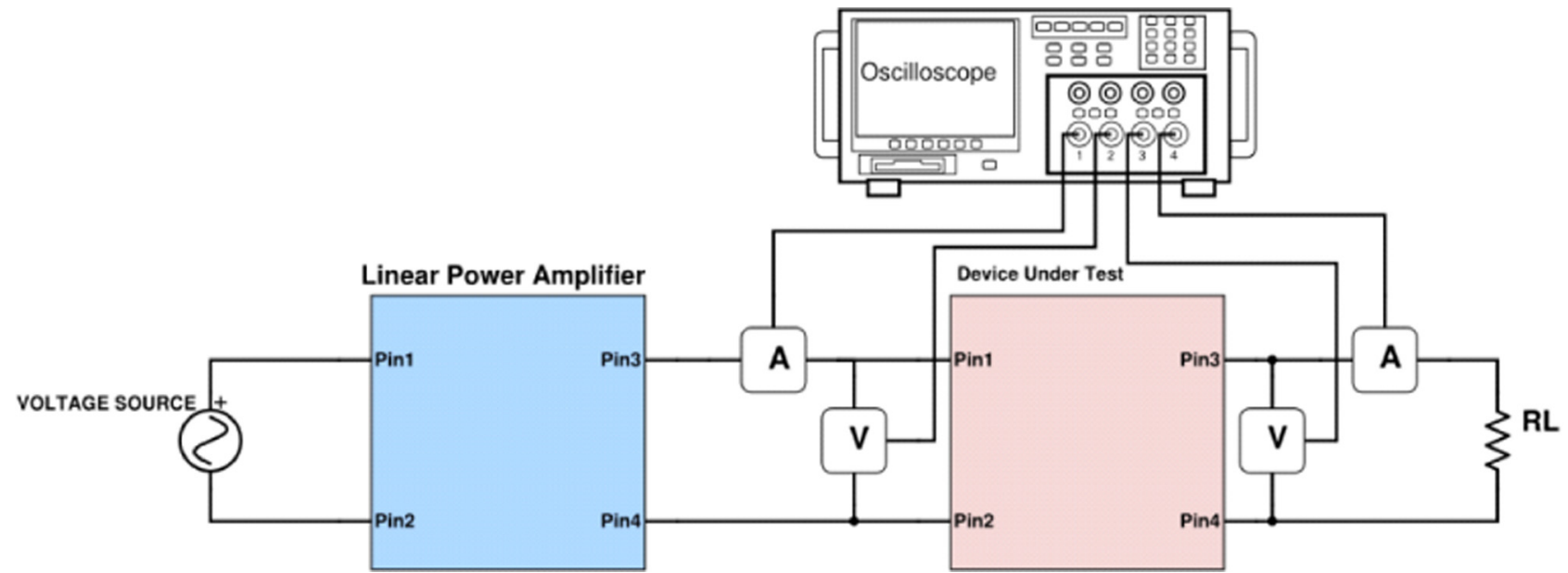

Fig. 19. The scheme of the experimental setup for the assessment of the resonant magnetic coupler performance. 

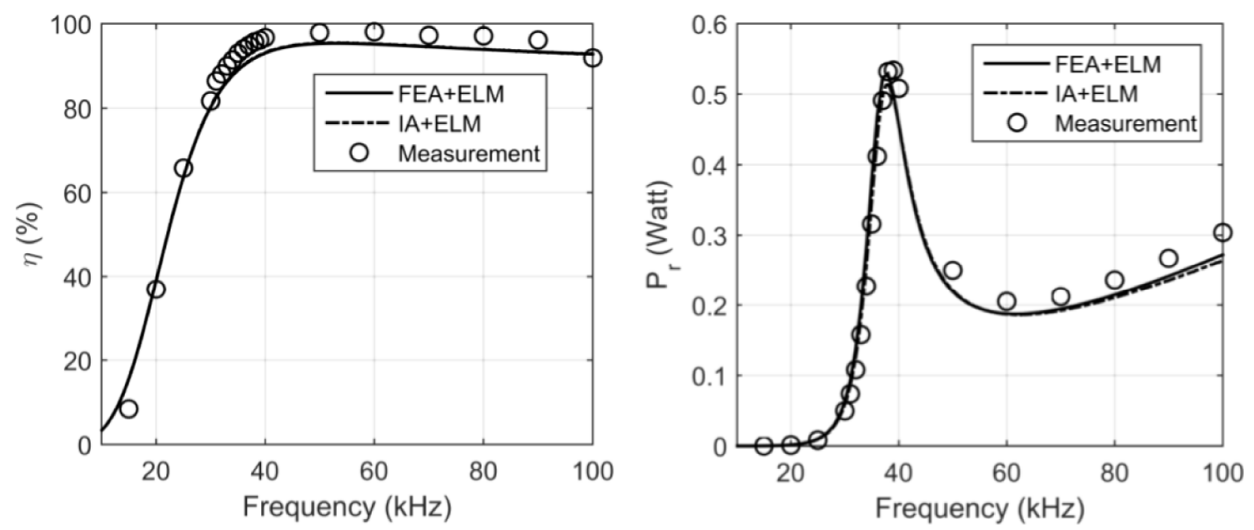

(a)
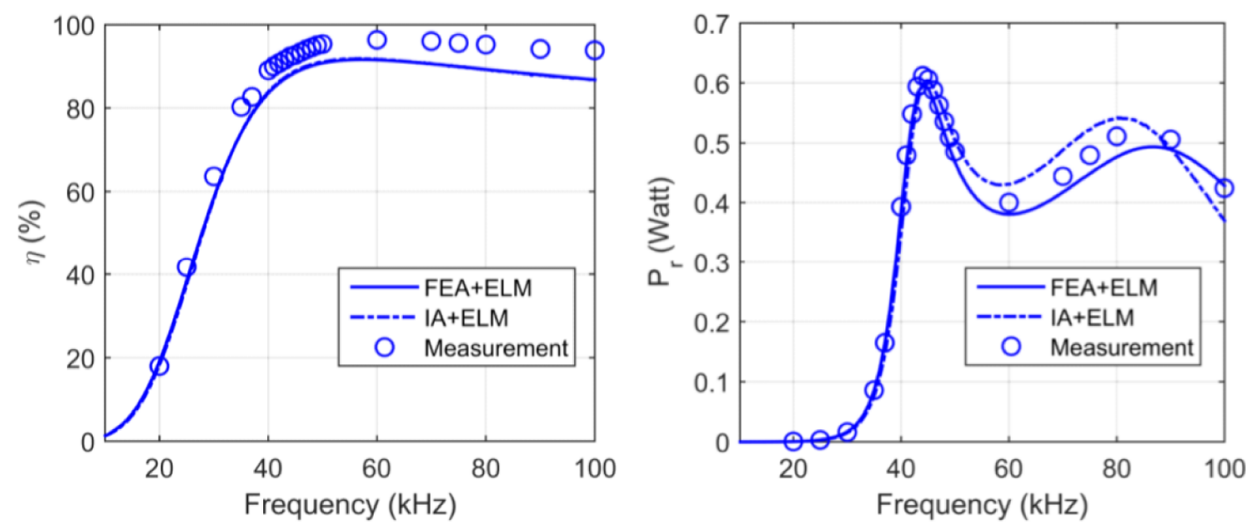

(b)
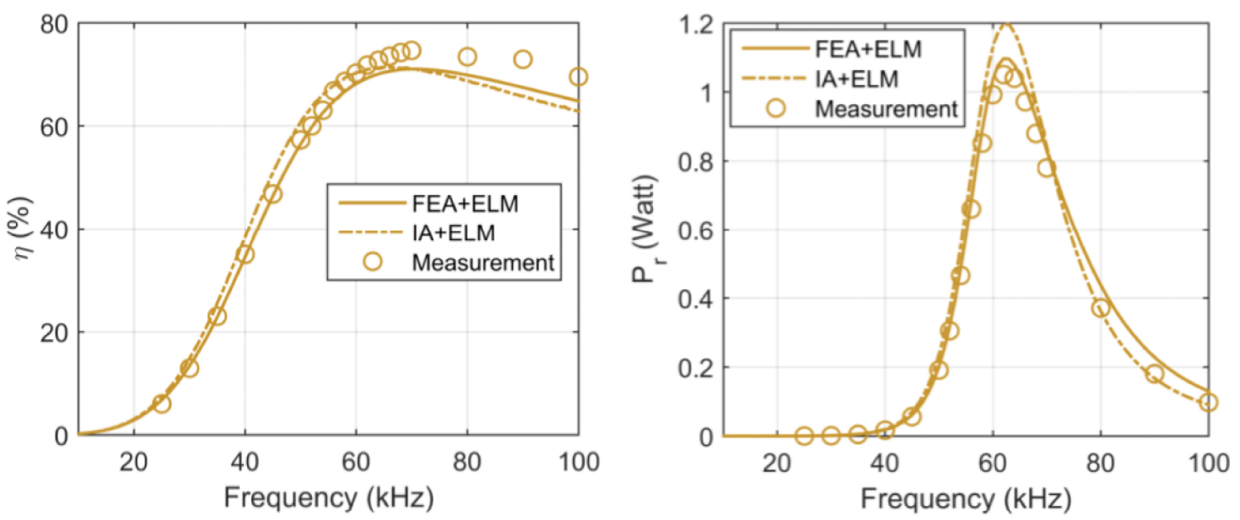

(c)

Fig. 20. System efficiency and received power as a function of the operating frequency for (a) $d=0 \mathrm{~mm},(\mathrm{~b}) d=10 \mathrm{~mm},(\mathrm{c}) d=20 \mathrm{~mm}$ with $f_{\text {res }}=50 \mathrm{kHz}, R_{L}=100 \Omega$ and $V_{\text {in }}=5$ Vpeak.

It is worth mentioning the concurrence of the simulation results and the measurement results shown in Figure 20. These experimental measurements on the prototype confirm the capability of the proposed design of the resonant magnetic coupling to achieve $\sim 500 \mathrm{~mW}$ at all distances with minimum efficiency of $\sim 70 \%$.

The characterization of the uncertainties of the experimental setup were carried out by connecting a resistor to the output of the linear power amplifier. This allowed the analysis of the accuracy of the phase angle measurement which is achieved by the voltage/current probes and the algorithm of the oscilloscope. The phase angle obtained for the resistor varies between $-0.1^{\circ}$ and $+0.1^{\circ}$ which is an accurate value for the assessment of our prototype. The accuracy of the voltage and current amplitude measurement is within $1 \mathrm{mV}$. Another source of uncertainty in our experimental test bench is the measurement of the distance between the coils, the error is assessed to be around $1 \mathrm{~mm}$.

Note that the aluminum housing in this design acts as shielding at the operating frequency (at $F=50 \mathrm{kHz}$ the skin depth in the aluminum tube is $0.356 \mathrm{~mm}$ ) which lead to an enhancement of the structure immunity to the electromagnetic interference. 


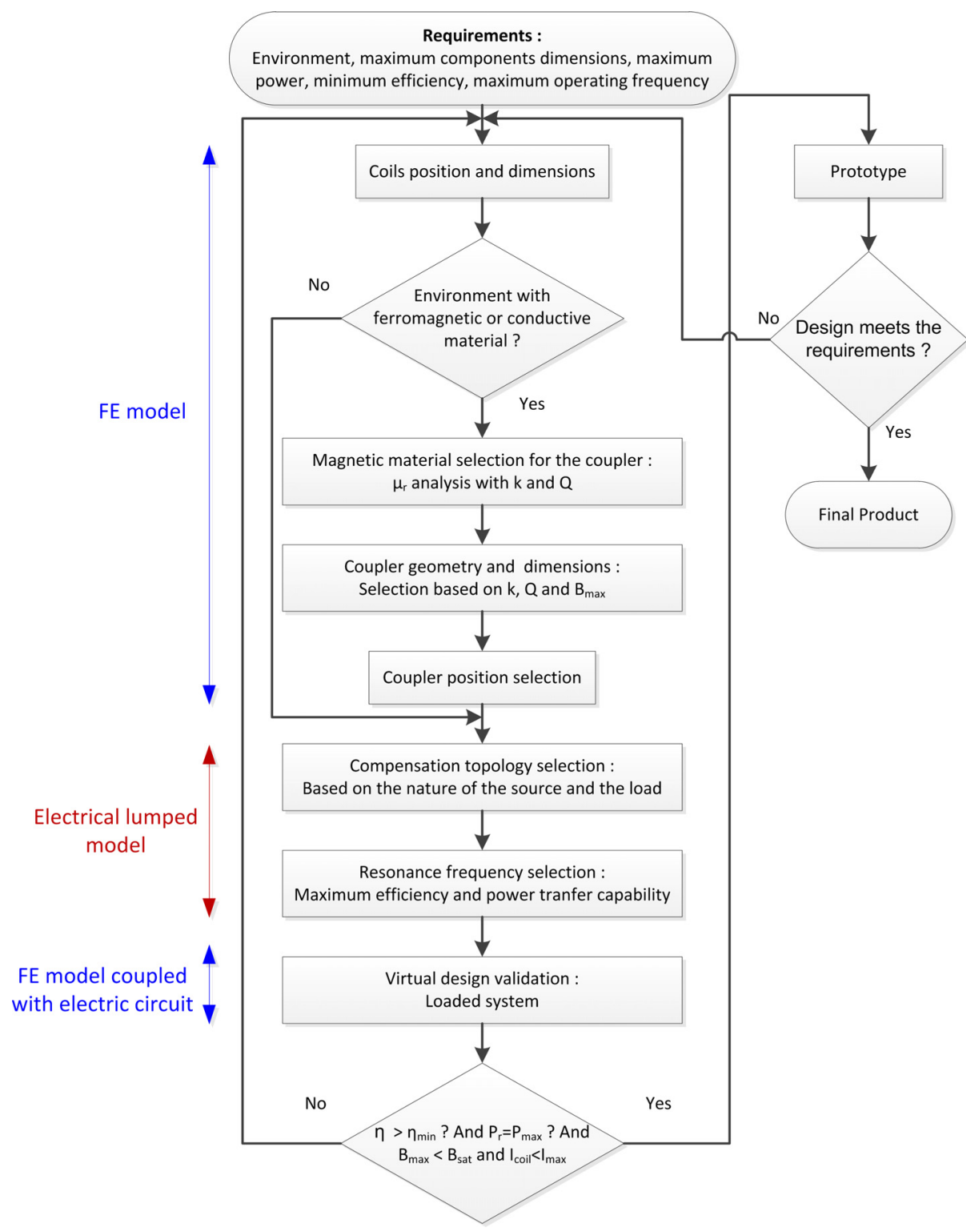

Fig. 21. Design methodology flowchart.

\subsection{Design methodology}

The design methodology flowchart shown in Figure 21 was adopted to design the resonant magnetic coupler presented in the previous section. The methodology starts with collecting the relevant requirements and ends with the assessment of the system performance relying on the combination of two simplified models: a FE model and an electrical lumped model at different stages of the design.

\section{Conclusion}

This paper proposes a methodology to design wireless power supply system for moving low power devices (e.g. sensors) operating in harsh electromagnetic environment. The presence of ferromagnetic and conductive materials leads to decrease the magnetic coupling between the coils and the system efficiency according to the simulation and experimental results. The performance of the system was enhanced by introducing a new structure based on tubes made of SFM to the initial structure of the application. The proposed resonant magnetic coupler design allows up to $75 \%$ efficiency at the maximum studied distance. The circuit analyses show that the resonance frequency for which the capacitors are tuned, the operating frequency and the load are key elements to optimize the device performance once the magnetic coupler is designed. An experimental setup with the whole DC-to-DC conversion chain shows an efficiency of $25 \%$ while receiving $\sim 500 \mathrm{~mW} 12.5$ VDC across a resistance load. The low efficiency is due to the fact that the input impedance of the power electronics at the receiver side does not match the optimal load of the magnetic coupler.

The wireless power transfer system could be utilized as a position sensor [20]. When the system is carefully designed to avoid the bifurcation phenomena, the position of the moving 
bar could be measured by simply tracking the resonance frequency that shifts with the variation of the magnetic coupling between the transmitter and the receiver coil.

\section{References}

1. N.M. Roscoe, M.D. Judd, Harvesting energy from magnetic fields to power condition monitoring sensors, IEEE Sensors J 13, $2263(2013)$

2. J.A. van Schalkwyk, G.P. Hancke, Energy harvesting for wireless sensors from electromagnetic fields around overhead power lines, in IEEE International Symposium on Industrial Electronics (ISIE), May, 2012, pp. 1128-1135

3. S. Pobering, N. Schwesinger, Power supply for wireless sensor systems, in 2008 IEEE Sensors, October, 2008, pp. 685-688

4. G.P. Hancke, N.A. Vorster, The feasibility of using resonant inductive power transfer to recharge wireless sensor network nodes, in 2014 IEEE Wireless Power Transfer Conference (WPTC), May, 2014, pp. 100-105

5. L. Angrisani, F. Bonavolontà, G. d'Alessandro, M. D'Arco, Inductive power transmission for wireless sensor networks supply, in 2014 IEEE Workshop on Environmental Energy and Structural Monitoring Systems (EESMS), September, 2014, pp. $1-5$

6. R. Trevisan, A. Costanzo, Wireless sensing and power transfer in a rotary tool, in 2015 IEEE MTT-S International Microwave Symposium, May, 2015, pp. 1-4

7. H. Dzapo, Z. Stare, N. Bobanac, Digital measuring system for monitoring motor shaft parameters on ships, IEEE Trans. Instrum. Measur. 58, 3702 (2009)

8. S. Ditze, A. Endruschat, T. Schriefer, A. Rosskopf, T. Heckel, Inductive power transfer system with a rotary transformer for contactless energy transfer on rotating applications, in 2016 IEEE International Symposium on Circuits and Systems (ISCAS), May, 2016, pp. 1622-1625

9. H. Sakamoto, K. Harada, S. Washimiya, K. Takehara, Y. Matsuo, F. Nakao, Large air-gap coupler for inductive charger [for electric vehicles], IEEE Trans. Magn. 35, 3526 (1999)

10. C.-S. Wang, G. Covic, O. Stielau, Power transfer capability and bifurcation phenomena of loosely coupled inductive power transfer systems, IEEE Trans. Ind. Electron. 51, 148 (2004)
11. Q. Chen, L. Jiang, J. Hou, X. Ren, X. Ruan, Research on bidirectional contactless resonant converter for energy charging between EVs, in IECON 2013-39th Annual Conference of the IEEE Industrial Electronics Society, November, 2013, pp. 1236-1241

12. J. Hou, Q. Chen, K. Yan, X. Ren, S.-C. Wong, C. Tse, Analysis and control of $\mathrm{s} / \mathrm{sp}$ compensation contactless resonant converter with constant voltage gain, in 2013 IEEE Energy Conversion Congress and Exposition (ECCE), September, 2013, pp. 2552-2558

13. K.K. Ean, B.T. Chuan, T. Imura, Y. Hori, Novel band-pass filter model for multi-receiver wireless power transfer via magnetic resonance coupling and power division, in 2012 IEEE 13th Annual Wireless and Microwave Technology Conference (WAMICON), April, 2012, pp. 1-6

14. Z. Pantic, S. Bai, S. Lukic, Zcs lcc - compensated resonant inverter for inductive-power-transfer application, IEEE Trans. Ind. Electron 58, 3500 (2011)

15. J. Kim, H.-C. Son, K.-H. Kim, Y.-J. Park, Efficiency analysis of magnetic resonance wireless power transfer with intermediate resonant coil, IEEE Antennas Wirel. Propag. Lett. 10, 389 (2011)

16. F. Zhang, X. Liu, S.A. Hackworth, R.J. Sclabassi, M. Sun, In vitro and in vivo studies on wireless powering of medical sensors and implantable devices, in IEEE/NIH Life Science Systems and Applications Workshop (LiSSA 2009), April, 2009, pp. 84-87

17. J.I. Agbinya, Wireless power transfer (River Publishers, Wharton, TX, USA, 2012)

18. R. Bosshard, J. Muhlethaler, J. Kolar, I. Stevanovic, Optimized magnetic design for inductive power transfer coils, in 2013 Twenty-Eighth Annual IEEE Applied Power Electronics Conference and Exposition (APEC), March, 2013, pp. 1812-1819

19. W. Liu, H. Tang, Analysis of voltage source inductive coupled power transfer systems based on zero phase angle resonant control method, in Second IEEE Conference on Industrial Electronics and Applications (ICIEA 2007), May, 2007, pp. 1873-1877

20. Y. Bu, M. Nishiyama, T. Ueda, Y. Tashima, T. Mizuno, Examination of wireless power transfer combined with the utilization of distance detection, IEEE Trans. Magn. 50, 1 $(2014)$

Cite this article as: Salaheddine Safour, Yves Bernard, Theoretical and experimental study of a wireless power supply system for moving low power devices in ferromagnetic and conductive medium, Eur. Phys. J. Appl. Phys. 80, 10903 (2017) 